\title{
Medical Image of the Week: Cheyne Stokes Breathing on Polysomnography
}

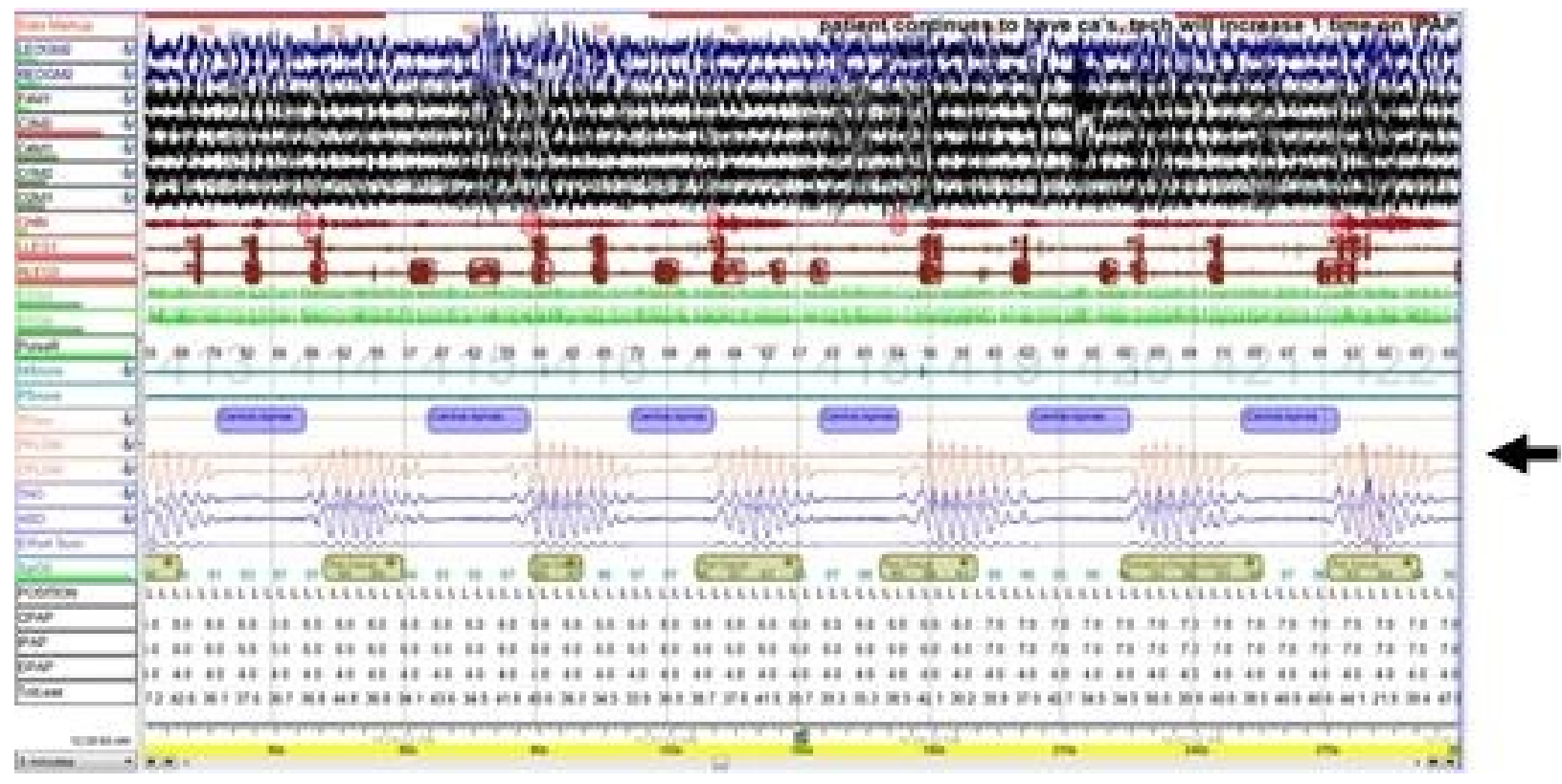

Figure 1. A 5-minute epoch showing Cheyne-Stokes breathing (arrow).

A 79-year-old man presented to the sleep lab for a split-night polysomnography (PSG) after a positive Berlin Questionnaire. He was screened and directly referred to our sleep lab through his PCP. Patient has a chart documented medical history of atrial fibrillation, idiopathic pulmonary fibrosis, obesity, and CHF. He did not have an echocardiogram available therefore the etiology of his CHF was unclear. He was found to have severe obstructive sleep apnea and was split early in the night. Prior to positive airway pressure, his apnea-hypopnea index (AHI) was 77 and were predominantly obstructive hypopneas. Soon after initiation of positive airway pressure, his PSG revealed the breathing pattern seen in Figure 1 . His respirations exhibited a crescendodecrescendo pattern (arrow) followed by a period of central apnea consistent with Cheyne Stokes breathing (CSB). In this patient, CSB was likely due to heart failure, although systolic or diastolic remained unclear. Of note, he was not on medications found to be responsible for CSB, and did not have a history of cerebral vascular accident.

Cheyne-Stokes breathing (CSB) is a well-documented but poorly understood abnormal breathing pattern that is believed to be a type of central sleep apnea (CSA), meaning apneas without upper airway obstruction. This compensatory mechanism is characterized by a cyclic change from oscillating events of apnea and hyperpnea. The characteristic feature of CSA-CSB is a longer cycle length, typically 45-60 seconds, alternating with a respiratory phase exhibiting a crescendo-decrescendo pattern of flow. This result is thought to be due to a delay in correction centrally when an elevated arterial PCO2 is detected within the blood stream by chemoreceptors. Co-morbid conditions often include cardiac disease (primarily heart failure independent of NYHA classification), neurologic disorders, prematurity, or sedation. Diagnosis is made by 
polysomnography. Treatment often entails treating the underlying cause or associated disorder. When all other strategies fail, remaining treatment includes the use of nocturnal continuous positive airway pressure (CPAP), supplemental oxygen, or adaptive servoventilation (ASV). Although, systolic heart failure with LVEF $<45 \%$ in patients with predominantly central sleep apnea currently precludes the use of ASV.

Tam Le, MD and Sekhon Kawanjit, MD Banner University Medical Center Tucson Tucson, AZ USA

\section{References}

1. Cherniack NS, Longobardo GS. Cheyne-Stokes breathing. An instability in physiologic control. N Engl J Med. 1973 May 3;288(18):952-7. [CrossRef] [PubMed]

2. Naughton M, Benard D, Tam A, Rutherford R, Bradley TD. Role of hyperventilation in the pathogenesis of central sleep apneas in patients with congestive heart failure. Am Rev Respir Dis. 1993 Aug;148(2):330-8. [CrossRef] [PubMed]

3. American Academy of Sleep Medicine. International classification of sleep disorders, 3rd ed. Darien, IL: American Academy of Sleep Medicine, 2014. 